\title{
Comparison of the Effectiveness of Haloperidol Injection and Oral Quetiapine to Control Delirium in Patients in the Emergency Department and Intensive Care Unit - A Randomized Clinical Trial
}

\author{
Morteza Talebi Doluee ${ }^{1}$, Zahra Abbasi Shaye², Mahdi Talebi ${ }^{3}$ and Abbas Farhadi' ${ }^{4, *}$ \\ ${ }^{1}$ Department of Emergency Medicine, Faculty of Medicine, Mashhad University of Medical Sciences, Mashhad, Iran \\ 2 Clinical Research and Development Unit of Akbar Hospital, Faculty of Medicine, Mashhad University of Medical Sciences, Mashhad, Iran \\ ${ }^{3}$ Assistant professor of Psychiatry, Department of Family Medicine, Mashhad University of Medical Sciences, Mashhad, Iran \\ ${ }^{4}$ Resident of Emergency Medicine, Faculty of Medicine, Mashhad University of Medical Sciences, Mashhad, Iran
}

* Corresponding author: Abbas Farhadi, Faculty of Medicine, Mashhad University of Medical Sciences, Mashhad, Iran. Tel: 09368535074; Email: Biomedical.sciences90@gmail.com

Received 2020 November 18; Revised 2020 December 22; Accepted 2021 January 06.

\begin{abstract}
Background: Delirium is a psychiatric syndrome observed among patients with critical psychiatric disorders. Haloperidol is now one of the first-line drugs for the treatment of delirium. However, quetiapine can be considered as an appropriate substitute in patients with a high risk of extrapyramidal symptoms or long QT syndrome.

Objectives: This study aimed to compare the effectiveness of intramuscular haloperidol and oral quetiapine to control delirium in patients in the emergency department and intensive care unit.

Methods: This randomized clinical trial was performed during 2017-2018 on patients with delirium who were referred to the emergency department and intensive care unit of Imam Reza Hospital (referral center), Mashhad, Iran. Patients were divided into two groups of 100 patients per group through a random allocation technique. In this study, $5 \mathrm{mg}$ of intramuscular haloperidol every 12 hours and $25 \mathrm{mg}$ of oral quetiapine were prescribed daily for the patients in the control and intervention groups, respectively. The delirium severity score of each patient was evaluated before and three days after the intervention through Delirium Rating Scale-Revised-98.

Results: In this study, the mean \pm SD age of participants was $60.2 \pm 14.1$ years. The findings indicated that no significant difference was observed between haloperidol $(22.7 \pm 1.9)$ and quetiapine $(22.7 \pm 2.2)$ groups in terms of the baseline delirium severity score $(\mathrm{P}=0.95)$. The mean delirium severity scores of patients in haloperidol and quetiapine groups were $25.6 \pm 2.1$ and $25.2 \pm 2.5$, respectively. Based on the obtained results, the difference between the delirium severity scores of both groups was not statistically significant $(\mathrm{P}=0.24)$.

Conclusion: Based on the results, oral quetiapine has a similar effect as intramuscular haloperidol and can be used as a substitute to this medicine for controlling the symptoms of patients with delirium.
\end{abstract}

Keywords: Delirium, Intramuscular haloperidol, Oral quetiapine

\section{Background}

Delirium is a common neuropsychology condition that is observed in critically ill patients with psychiatric disease (1). According to DSMV-TR, Delirium is a consciousness and cognitive disorder that develops over a short period of time and leads to a range of different clinical features, such as 1:altered level of consciousness, 2:changes in attention such as poor concentration and memory, 3:disorientation, 4: Rapid onset of the disease within a few hours to a few days, 5:a disease with a short period, 6:having a significant fluctuation that increases overnight (1). It is worth mentioning that cognitive impairment, movement disorders, and sleep disorders are among other features of delirium. The incidence of delirium in admitted patients is associated with an increase in the mortality rate, hospitalization, costs, and reduction in the rehabilitation of patient (1-4). Studies have shown that $10 \%-40 \%$ of elderly patients suffer from delirium during the course of hospitalization. According to some studies, the prevalence rate of delirium was $10 \%-18 \%$ among patients who were hospitalized due to surgical or non-surgical treatments, which was associated with higher mortality and morbidity rate (2).

Delirium may be associated with mental illnesses, such as bipolar, personality, generalized anxiety, panic, and major depressive disorders, as well as schizophrenia. Delirium may also be associated with diseases of the central nervous system, such as Parkinson's, Alzheimer's, and other mental disorders, and observed with a wide range of medical diseases, such as thyrotoxicosis, encephalitis, meningitis, hypoglycemia, uremic and hepatic encephalopathy, and head trauma. Patients with substance (e.g., alcohol, cocaine, and methamphetamine) dependence may experience delirium as well (3). Therefore, this condition is observed in a wide range of patients who are referred to the emergency department.

In the last decade, first-generation antipsychotics and benzodiazepines were used to control delirium. The most common first-line antipsychotic drug used in patients with delirium was haloperidol which was used due to the patients 'desire for dopamine, low anticholinergic side effects, and various routes of 
administration. However, haloperidol and other generations of antipsychotics cause various complications, such as extrapyramidal syndrome, which has led to a reduction in the use of these drugs (1). The application of second-generation antipsychotics showed that they can be used to control delirium in patients with agitation in the emergency departments and that these medicines do not cause such side effects as extrapyramidal complications, hypotension, and excessive sleeplessness associated with the use of haloperidol. Therefore, the tendency to use these drugs for the control of delirium has increased considerably in recent years (4).

Further medical studies were conducted to determine the best medicine for the control of delirium. Therefore, a wide range of medications was investigated for this purpose, including quetiapine, which is a second-generation antipsychotic medicine. Quetiapine is available on the market as tablets and is effective against psychotic symptoms. It is worth mentioning that, the incidence of quetiapine's extrapyramidal side effects is very low. Quetiapine has a linear pharmacokinetic and quickly reaches its peak plasma concentration (90 minutes in the fastacting oral form versus 10 to 20 minutes in intramuscular haloperidol). This medicine is an agonist of histamine and adrenergic receptors and often causes somnolence in patients which is less severe than that caused by the first generation medicines (5). A few other types of studies were performed to evaluate the management of delirium in recent years, including reports (6-10), retrospective researches (11), randomized controlled trials, (12$14)$, and open-label trials (15-21). These studies showed that quetiapine was more effective than placebo and as effective as haloperidol $(13,14)$ in the control of delirium (15). Therefore, it seems that quetiapine can control delirium symptoms in patients hospitalized in the emergency department and intensive care unit (ICU). However, the number of studies conducted in this field and the patients treated by quetiapine $(n>200)$ are limited. Moreover, due to the limited number of reported results, it is not possible to conclude that quetiapine is more effective than haloperidol in the control of delirium and further studies need to be done in this regard.

\section{Objectives}

The present study aimed to compare the effectiveness of haloperidol injection and oral quetiapine in the control of delirium in the patients admitted in the emergency department and ICU.

\section{Methods}

\subsection{Participants}

This randomized equivalence clinical trial was performed on delirium patients who were referred to the emergency department and ICU of Imam Reza Hospital, Mashhad, Iran, from March 2017 to 2018. Imam Reza educational and treatment center is a tertiary referral center in Razavi Khorasan Province, Iran.

The study population included patients aged 18 and over whose delirium condition was confirmed based on the Diagnostic and Statistical Manual of Mental Disorders, $5^{\text {th }}$ edition, and psychiatric consulting. On the other hand, the patients with dementia, poisoning, alcohol and benzodiazepines deprivation, visual, and hearing impairment, lack of response to verbal or physical stimuli, history of long QTc $(>500 \mathrm{~ms})$, history of medicine allergy, neuroleptic malignant syndrome, Parkinson's, mental diseases, and mood disorders were excluded from the study.

\subsection{Sample size}

The sample size was calculated based on a previous clinical trial study (22), using the NonInferiority/equivalence Tests for the Difference Between Two Means (delirium severity scores in haloperidol and quetiapine groups were estimated at $11.46 \pm 6.58$ and $9.41 \pm 7.29$, respectively) considering $\alpha=0.05$ and $1-\beta=0.8$ for power analysis and sample size (version 11). The sample size was calculated at 96 cases per group; however, regarding the attrition rate of $5 \%, 200$ patients were included in the study that were divided into two groups (100 cases per group) through a random sampling method.

\subsection{Ethics}

The study protocol was approved by the Ethics Committee of the Mashhad University of Medical Sciences, Iran, (IR.MUMS.fm.REC.1396.599). The written informed consent was obtained from the participants' first-degree relatives or legal guardians and details were fully explained to them prior to the beginning of the study. Afterward, the patients were randomly divided (through the computer-generated random assignment) into two groups. Through the allocation concealment process, the randomized codes of the groups were placed in the sealed envelopes. Therefore the physicians and researchers were blinded to the assigned treatments for each group in this study.

\subsection{Intervention}

Regarding the frequency of delirium symptoms at the end of the day, $5 \mathrm{mg}$ intramuscular haloperidol was injected every 12 hours to patients in the first group (at 8:00 and 20:00 o'clock), and $25 \mathrm{mg}$ oral quetiapine was given daily to the patients in the second group. A physician adjusted the dosage based on the clinical evaluation during the study. During the treatment period, patients received medications related to clinical conditions and underlying diseases, 
and the treatment team and ICU personnel were asked to avoid sensory stimulation for patients.

\subsection{Outcome}

The demographic characteristics form, including information about the cause of admission, and underlying diseases was completed by a resident researcher who was blinded to the patient's assigned group. The delirium severity of each patient was evaluated four times (before, one, two, and three days after the intervention) through the Delirium Rating Scale-Revised-98 scoring system. It should be mentioned that the scoring system consisted of 13 items related to delirium severity. These items covered information about sleep disorders, a disorder of perception, delirium, affection, language, thought disorders, restless movements, slow movements, orientation, concentration, short-term memory, long-term memory, and spatial visualization ability. Each item received a score from 0 to 3 based on the severity of the symptoms. In the evaluation of the symptoms severity, the highest and lowest scores were 39 and 0 , respectively. All evaluations were performed from 18:00 to 20:00 o'clock.

To analyze the obtained results, intention-to-treat analysis was used by including the data gathered from all randomized patients who received at least one dose of medication and were assessed at least once after random assignment.

\subsection{Statistical analysis}

The data were analyzed using SPSS software (version 11) through the Kolmogorov-Smirnov test. Descriptive statistics were presented as tables and charts. The analysis was done based on the patients' intention to be treated. Independent t-test and Chisquare test were used to compare quantitative variables. Wilcoxon analysis and Friedman test were utilized to evaluate the scores before and after using the medicine and to calculate the changes in delirium severity during the time in both haloperidol and quetiapine groups.

\section{Results}

This study included 200 patients (100 cases per group) with delirium who had met the inclusion criteria (in total 30 patients were excluded from the study for having Parkinson's disease $(n=2)$ and long QTc $(n=3)$; being drug poisoned $(n=5)$; and not providing written informed consent $(n=20$, Figure 1$)$.

The mean \pm SD age of the patients was $60.1 \pm 14.1$

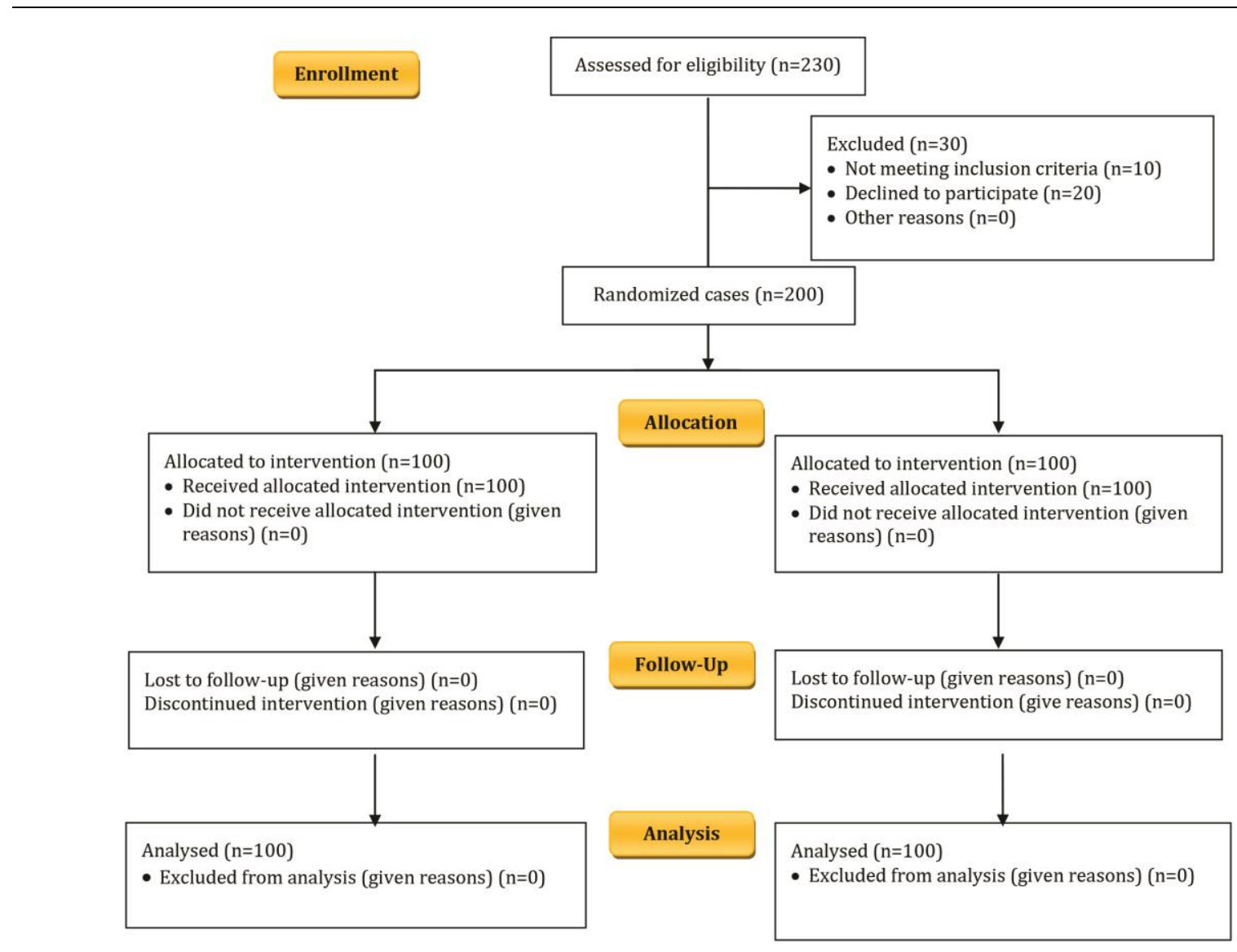

Figure 1. CONSORT 2010 flow diagram 


\begin{tabular}{|c|c|c|c|c|}
\hline Variable & & Quetiapine group & Haloperidol group & P-value \\
\hline \multirow{4}{*}{$\begin{array}{l}\text { Underlying } \\
\text { disease }\end{array}$} & stroke & $5(2.5 \%)$ & $8(4 \%)$ & $0.230^{*}$ \\
\hline & Metabolic & $38(19 \%)$ & $29(14.5 \%)$ & $0.398^{*}$ \\
\hline & Infectious & $48(26 \%)$ & $51(25.5 \%)$ & $0.987^{*}$ \\
\hline & Cardiovascular & $9(5.5 \%)$ & $12(6 \%)$ & $0.678^{*}$ \\
\hline Gender & Male & $62(62 \%)$ & $63(63 \%)$ & 0.888 \\
\hline Age & Female & $\begin{array}{l}38(38 \%) \\
61.1 \pm 14.1\end{array}$ & $\begin{array}{c}37(37 \%) \\
59.3 \pm 14\end{array}$ & $0.37 * *$ \\
\hline
\end{tabular}

**: T-Test analysis

years (the age range of 53-76 years). Moreover, the majority of the patients were male $(n=125,62.5 \%)$, and no significant difference was observed in the basic characteristics of the patients in both haloperidol and quetiapine groups (Table 1). Infectious diseases were the most common cause of admission ( $n=99,49.5 \%)$. Furthermore, there was not any statistically significant difference in terms of underlying diseases between the two groups $(\mathrm{P}=0.49)$.

Table 2 presents the total score of the severity of symptoms before the treatment and on the first, second, and third day after starting the treatment in both haloperidol and quetiapine groups. No statistically significant difference was observed between the two groups.

Comparison of the severity of symptoms before and after the start of the treatment in the haloperidol group showed a statistically significant difference in the first $(\mathrm{P}=0.01)$, second $(\mathrm{P}<0.001)$, and third day $(\mathrm{P}<0.001)$ after the start of the treatment (Table 3$)$. In addition, there were statistically significant differences between the scores of the severity of symptoms in the quetiapine group before the treatment and those on the first $(\mathrm{P}=0.01)$, second $(\mathrm{P}<0.001)$, and the third day $(\mathrm{P}<0.001)$ after the treatment (Table 3).

Friedman test showed that there was a significant difference in the scores of the severity of symptoms in haloperidol and quetiapine groups $(\mathrm{P}=0.001)$ during the time; however, no significant difference was observed between the haloperidol and quetiapine groups in terms of the severity of the symptoms over time $(\mathrm{P}=0.513)$.

\begin{tabular}{lcc}
\hline $\begin{array}{l}\text { Table 2. Total score of the severity of symptoms before the treatment and on the first, second, and third day after the treatment in both } \\
\text { haloperidol and quetiapine groups }\end{array}$ & Haloperidol & Quetiapine \\
\hline & $25.6 \pm 2.1$ & $25.2 \pm 2.5$ \\
\hline Pean of total score & $22.7 \pm 1.9$ & $22.7 \pm 2.2$ \\
Severity of symptoms before the treatment & $20.9 \pm 2.2$ & $20.6 \pm 2.1$ \\
Severity of the symptoms on the first day after the treatment & $16.7 \pm 1.5$ & $16.8 \pm 1.9$ \\
Severity of symptoms on the second day after the treatment & $8.5 \pm 1.3$ & 0.946 \\
Severity of symptoms on the third day after the treatment & 0.251 & 0.752 \\
\hline
\end{tabular}

*: Man Whitney test

Table 3. Comparison of the symptom severity before the treatment on the first, second, and third day after the start of the treatment in the quetiapine and haloperidol groups

\begin{tabular}{|c|c|c|c|c|}
\hline \multirow{2}{*}{$\begin{array}{l}\text { Severity score before the treatment (Quetiapine group) Mean } \pm \text { SD } \\
\text { Median (IQR)** }\end{array}$} & \multicolumn{3}{|c|}{ Severity score after treatment } & \multirow[b]{2}{*}{ P-Value* } \\
\hline & Day & Mean \pm SD & Median (IQR) & \\
\hline \multirow{3}{*}{$\begin{array}{l}22.7 \pm 2.2 \\
22(21-25)\end{array}$} & First day & $20.6 \pm 2.1$ & $21(19-22)$ & 0.01 \\
\hline & Second day & $16.8 \pm 1.9$ & $17(16-18)$ & $<0.001$ \\
\hline & Third day & $8.4 \pm 1.2$ & $9(8-9)$ & $<0.001$ \\
\hline \multirow{2}{*}{$\begin{array}{l}\text { Severity score before the treatment (Haloperidol group) Mean } \pm \text { SD } \\
\text { Median (IQR) }\end{array}$} & \multicolumn{3}{|c|}{ Severity score after treatment } & P-Value \\
\hline & Day & Mean \pm SD & Median (IQR) & P-Value \\
\hline \multirow{3}{*}{$\begin{array}{l}22.7 \pm 1.9 \\
22(21-24)\end{array}$} & First day & $20.9 \pm 2.2$ & $20(19-21)$ & 0.01 \\
\hline & Second day & $16.7 \pm 1.5$ & $16(15-17.75)$ & $<0.001$ \\
\hline & Third day & $8.5 \pm 1.3$ & $8(8-9)$ & $<0.001$ \\
\hline
\end{tabular}

**: Inter quartile range

\section{Discussion}

Delirium is a psychiatric syndrome in patients with underlying diseases. Treatment for this condition requires the treatment of underlying diseases and the improvement of patients' orientation and physical functions. The effect of antipsychotics on the treatment of delirium has been studied during the last two decades. The present study is an important step in examining this field.

The results of this study indicated that there was no significant difference between quetiapine and haloperidol in controlling the symptoms of delirium and that both medicines were equally effective in this 
regard. Moreover, based on the results, quetiapine has delayed peak plasma concentration, compared to haloperidol $(90 \mathrm{~min}$ with the fast-acting oral form of quetiapine versus 10 to $20 \mathrm{~min}$ with intramuscular haloperidol). However, it should be noted that the oral consumption of quetiapine is more safe and easy for the patients, compared to the intramuscular haloperidol that must be used along with midazolam.

In the same line, the study conducted by Yoon (2013) in Korea investigated and compared the effects of haloperidol with those of risperidone, olanzapine, and quetiapine in patients with delirium for six days. The results showed that all four drugs used in the study improved delirium symptoms; however, antipsychotics should be taken with caution in elderly patients (23). Another study conducted by Grover (2016) in India compared the effects of haloperidol and quetiapine in patients with delirium and demonstrated that the mean delirium Rating Scale-Revised-98 scores were not significantly different after the treatment (22). The results of another study conducted by Hawkins (2013) showed that the effects of quetiapine on the treatment of delirium reduced the duration of treatment in patients, regardless of the applied doses. Quetiapine showed effects similar to haloperidol and had no side effects (24). Similarly, the study performed by Maneeton showed that low-dose quetiapine and haloperidol may be equally effective and safe for controlling delirium symptoms (25).

Furthermore, the results of the studies conducted by Rod (2015) and Modbernya (2002) revealed that delirium syndrome was observed with a high frequency in ICU and that older people were at higher risk of developing the syndrome. The present study was conducted on 200 patients with delirium and a mean \pm SD age of $60.1 \pm 14.1$ years. Delirium prevalence in the present study was consistent with that in both previously mentioned studies in patients with high mean age. In the study conducted by Yoon and Grover, the mean \pm SD age of patients was estimated at $11.5 \pm 71$ and 46 years, respectively (23). It should be noted that the number of patients in the Grover study was 63 cases (22). The aforementioned studies were similar to the present study, however, they were conducted in various centers. In this study, samples were collected from referral and general centers.

The applied doses of quetiapine in the Grover study (2016), which was conducted in India, were estimated at 12.5 to $75 \mathrm{mg}$ (22). The results of the Maneeton study (2013) in Thailand showed that 25$100 \mathrm{mg}$ quetiapine produced similar effects as haloperidol in controlling delirium symptoms in patients (25). In the present study, $25 \mathrm{mg}$ dose of quetiapine was prescribed; therefore, it seems that the lower dose of medicine can control the delirium symptoms in the Iranian population.

In the study performed by Crawford (2013) in
Australia, the mean applied dose of haloperidol was $2.1 \mathrm{mg}$ per day, and one out of 15 patients discontinued haloperidol due to its side effects (26). The present study did not evaluate the complications of using these two medications. Therefore, it is suggested that future studies evaluate these complications.

In a recent study, quetiapine was used for delirium prophylaxis and the results revealed that the incidence of delirium was $45.5 \%$ and $77.6 \%$ in patients during the admission to ICU in the quetiapine group and the group that did not receive pharmacological prophylaxis, respectively. It is suggested that future studies investigate these results (27).

\subsection{Strengths and Limitation}

This study is one of the most comprehensive studies conducted to assess the effects of quetiapine on patients with delirium. The previous studies in this regard were performed on a limited number of patients.

Regarding the limitations of the present study, one can refer to the lack of the evaluation of haloperidol and quetiapine side effects and the lack of a placebo group to check the side effects of the medicine. Moreover, this study did not evaluate the effects of different types of delirium, including hypoactive delirium.

In a recent study, quetiapine prophylaxis was used to control delirium symptoms which can be the subject of future studies.

\section{Conclusion}

The results of the present study showed that the use of $25 \mathrm{mg}$ oral quetiapine per day had the same effect as $5 \mathrm{mg}$ haloperidol twice a day in controlling the symptoms of patients with delirium. Therefore, quetiapine can be used as alternative medicine in cases of haloperidol contraindications or restrictions.

\section{Acknowledgments}

This study is extracted from the specialty Ph.D. thesis, authored by Dr. Abbas Farhadi. The authors would like to thank and appreciate Mashhad university of Medical Sciences research committee for supporting the present study and the emergency department personnel of Edalatian and Hasheminezhad Hospitals, Mashhad, Iran for their cooperation in this study.

\section{Footnotes}

Authors' Contribution: Morteza Talebi Doluee: Designed and performed experiments.

Zahra Abbasi Shaye: Analyzed data and co-wrote the paper

Mahdi Talebi: Supervised the research 
Abbas Farhadi: Performed experiments and co-wrote the paper.

Conflict of Interests: All authors declare that there is no conflict of interests regarding the publication of the present study.

Ethical Approval: The study protocol was approved by the Ethics Committee of the Mashhad University of Medical Sciences, Iran.

Funding/Support: This study was funded by Mashhad University of Medical Sciences.

Informed consent: The written informed consent was obtained from the participants' first-degree relatives or legal guardians.

\section{References}

1. Lahariya S, Grover S, Bagga S, Sharma A. Phenomenology of delirium among patients admitted to a coronary care unit. Nord J Psychiatry. 2016;70(8):626-32. doi: 10.1080/08039488. 2016.1194467. [PubMed: 27329002].

2. Oh-Park M, Chen P, Romel-Nichols V, Hreha K, Boukrina O, Barrett AM. Delirium screening and management in inpatient rehabilitation facilities. Am J Phys Med Rehabil. 2018; 97(10):754-62. doi: 10.1097/PHM.0000000000000962. [PubMed: 29742533].

3. Ciampi A, Bai C, Dyachenko A, McCusker J, Cole MG, Belzile E. Latent class analysis of the multivariate Delirium Index in longterm care settings. Int Psychogeriatr. 2019;31(1):59-72. doi: 10.1017/S1041610218000510. [PubMed: 29720281].

4. Rengel KF, Pandharipande PP, Hughes CG. Postoperative delirium. Presse Med. 2018;47(4 Pt 2):e53-64. doi: 10.1016/ j.lpm.2018.03.012. [PubMed: 29680484].

5. Wang HR, Woo YS, Bahk WM. Atypical antipsychotics in the treatment of delirium. Psychiatry Clin Neurosci. 2013; 67(5):323-31. doi: 10.1111/pcn.12066. [PubMed: 23859663].

6. Torres R, Mittal D, Kennedy R. Use of quetiapine in delirium: case reports. Psychosomatics. 2001;42(4):347-9. doi: 10.1176/ appi.psy.42.4.347. [PubMed: 11496025].

7. 7. Al-Samarrai S, Dunn J, Newmark T, Gupta S. Quetiapine for treatment-resistant delirium. Psychosomatics. 2003;44(4):3501. doi: 10.1176/appi.psy.44.4.350. [PubMed: 12832605].

8. Kato D, Kawanishi C, Kishida I, Furuno T, Matsumura T, Hasegawa $\mathrm{H}$, et al. Delirium resolving upon switching from risperidone to quetiapine: implication of CYP2D6 genotype. Psychosomatics. 2005;46(4):374-5. doi: 10.1176/appi.psy.46.4.374. [PubMed: $16000684]$.

9. Seemüller F, Volkmer E, Vogel T, Hummel T, Krauseneck T, Riedel $\mathrm{M}$, et al. Quetiapine as treatment for delirium during weaning from ventilation: a case report. J Clin Psychopharmacol. 2007; 27(5):526-8. doi: 10.1097/JCP.0b013e3181513905. [PubMed: 17873696].

10. Almeida F, Albuquerque E, Murta I. Delirium induced by quetiapine and the potential role of norquetiapine. Front Neurosci. 2019;13:886. doi: 10.3389/fnins.2019.00886. [PubMed: 31481872].

11. Schwartz TL, Masand PS. Treatment of delirium with quetiapine. Prim Care Companion J Clin Psychiatry. 2000; 2(1):10-12. doi: 10.4088/pcc.v02n0103. [PubMed: 15014661].

12. Lee JY, Kim SH, Seo EG, Lee JY, Shim JY, Lee YH. Comparative study of haloperidol and quetiapine in the treatment of delirium: a prospective, open clinical trial. J Korean Soc Biol Ther Psychiatry. 2006;12:162-71. doi: 10.1016/S0924977X(06)70656-3.
13. Devlin JW, Roberts RJ, Fong JJ, Skrobik Y, Riker RR, Hill NS, et al. Efficacy and safety of quetiapine in critically ill patients with delirium: a prospective, multicenter, randomized, doubleblind, placebo-controlled pilot study. Crit Care Med. 2010; 38(2):419-27. doi: 10.1097/CCM.0b013e3181b9e302. [PubMed: 19915454].

14. Tahir TA, Eeles E, Karapareddy V, Muthuvelu P, Chapple S, Phillips B, et al. A randomized controlled trial of quetiapine versus placebo in the treatment of delirium. J Psychosom Res. 2010;69(5):485-90. doi: 10.1016/j.jpsychores.2010.05.006. [PubMed: 20955868].

15. Maneeton B, Maneeton N, Srisurapanont M. An open-label study of quetiapine for delirium. J Med Assoc Thai. 2007; 90(10):2158-63. [PubMed: 18041437].

16. Kim KY, Bader GM, Kotlyar V, Gropper D. Treatment of delirium in older adults with quetiapine. J Geriatr Psychiatry Neurol. 2003;16(1):29-31. doi: 10.1177/0891988702250533. [PubMed: 12641370].

17. Lee KU, Won WY, Lee HK, Kweon YS, Lee CT, Pae CU, et al. Amisulpride versus quetiapine for the treatment of delirium: a randomized, open prospective study. Int Clin Psychopharmacol. 2005;20(6):311-4. doi: 10.1097/00004850-200511000-00005 [PubMed: 16192839].

18. Shinno H, Matsuoka T, Yamamoto O, Noma $Y$, Hikasa $S$, Takebayashi M, et al. Successful treatment with quetiapine for delirium in terminally ill cancer patients. Psychogeriatrics. 2007;7(2):64-8. doi: 10.1111/j.1479-8301.2007.00182.x.

19. Omura K, Amano N. Clinical experience of quetiapine in 24 elderly patients with delirium. Psychogeriatrics. 2003;3(2):6972. doi: 10.1046/j.1479-8301.2003.00012.x.

20. Sasaki Y, Matsuyama T, Inoue S, Sunami T, Inoue T, Denda K, et al. A prospective, open-label, flexible-dose study of quetiapine in the treatment of delirium. J Clin Psychiatry. 2003; 64(11):1316-21. doi: 10.4088/jcp.v64n1106. [PubMed: $14658945]$.

21. Pae CU, Lee SJ, Lee CU, Lee C, Paik IH. A pilot trial of quetiapine for the treatment of patients with delirium. Hum Psychopharmacol. 2004;19(2):125-7. doi: 10.1002/hup.559. [PubMed: 14994323].

22. Grover S, Mahajan S, Chakrabarti S, Avasthi A. Comparative effectiveness of quetiapine and haloperidol in delirium: A single blind randomized controlled study. World J Psychiatry. 2016;6(3):365-71. doi: 10.5498/wjp.v6.i3.365. [PubMed: 27679777].

23. Yoon HJ, Park KM, Choi WJ, Choi SH, Park JY, Kim JJ, et al. Efficacy and safety of haloperidol versus atypical antipsychotic medications in the treatment of delirium. BMC Psychiatry. 2013;13:240. doi: 10.1186/1471-244X-13-240. [PubMed: 24074357].

24. Hawkins SB, Bucklin M, Muzyk AJ. Quetiapine for the treatment of delirium. J Hosp Med. 2013;8(4):215-20. doi: 10.1002/ jhm.2019. [PubMed: 23468358].

25. Maneeton B, Maneeton N, Srisurapanont M, Chittawatanarat K. Quetiapine versus haloperidol in the treatment of delirium: a double-blind, randomized, controlled trial. Drug Des Devel Ther. 2013;7:657-67. doi: 10.2147/DDDT.S45575. [PubMed: 23926422].

26. Crawford GB, Agar M M, Quinn SJ, Phillips J, Litster C, Michael $\mathrm{N}$, et al. Pharmacovigilance in hospice/palliative care: net effect of haloperidol for delirium. J Palliat Med. 2013; 16(11):1335-41. doi: 10.1089/jpm.2013.0230. [PubMed: 24138282].

27. Abraham MP, Hinds M, Tayidi I, Jeffcoach DR, Corder JM, Hamilton LA, et al. Quetiapine for delirium prophylaxis in highrisk critically ill patients. Surgeon. 2020;20:S1479. doi: 10.1016/j.surge.2020.02.002. [PubMed: 32213291]. 